\title{
Hand-foot syndrome related to chemotherapy
}

\author{
Jianjun Qiao MD PhD, Hong Fang MD Mphil
}

Competing interests: None declared.

This article has been peer reviewed.

Affiliation: From the Department of Dermatology, the First Affiliated Hospital, College of Medicine, Zhejiang University, China

Correspondence to: Hong Fang, fanghongzy@sina.com

CMAJ 2012. DOI:10.1503 /cmaj.111309

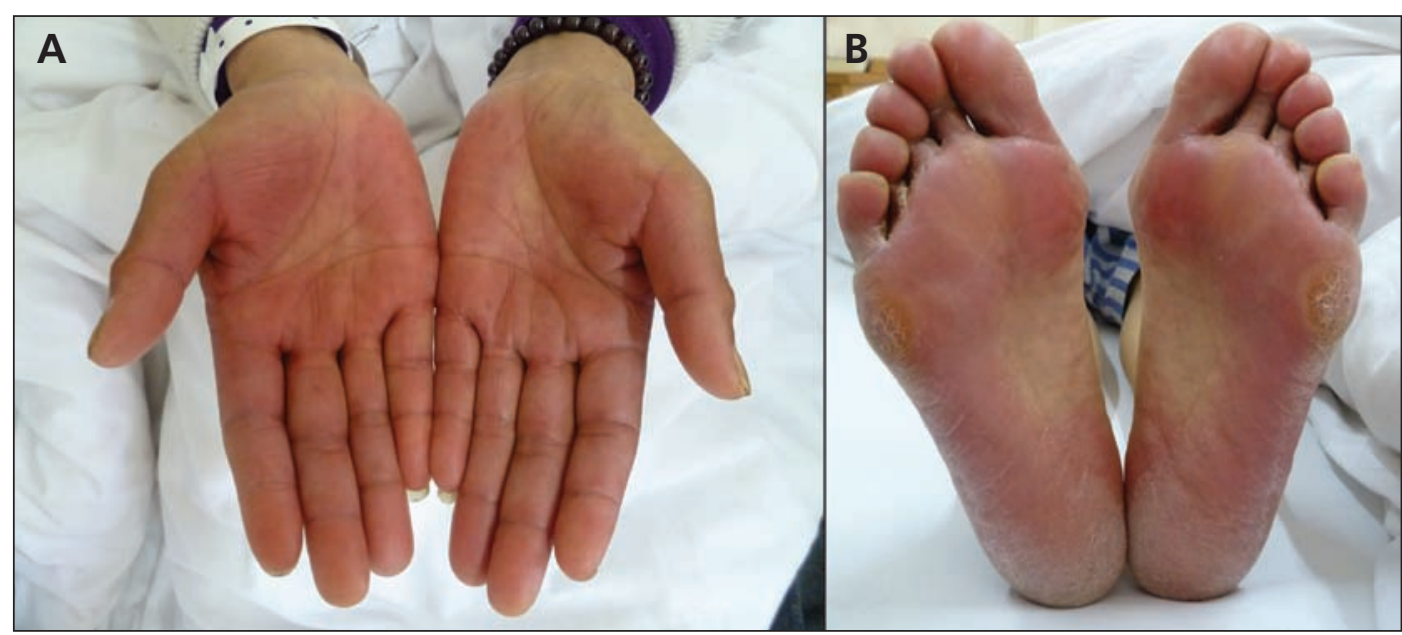

Figure 1: Sharply demarcated erythema of the palms (A) and soles (B) of a 59-year-old woman undergoing chemotherapy for rectal cancer.

A 59-year-old woman with advanced rectal cancer underwent a 2-week course of chemotherapy with capecitabine (on days 1-14) and oxaliplatin (on day 1). On day 9, she developed sharply demarcated erythema, swelling and pain of her palms and soles (Figure 1). The eruption progressed during the course of chemotherapy, and hand-foot syndrome associated with capecitabine was diagnosed. The erythema was treated with topical urea cream, and it completely resolved 10 days after the last dose of capecitabine.

Hand-foot syndrome (also known as handfoot reaction, palmar-plantar erythrodysesthesia, chemotherapy-associated acral erythema or Burgdorf reaction) is a cutaneous toxicity ${ }^{1-3}$ that is most frequently linked to cytotoxic drugs (e.g., 5-fluorouracil, capecitabine, cytarabine, docetaxel and pegylated liposomal doxorubicin) ${ }^{1}$ and multikinase-inhibitors (e.g., sorafenib and sunitinib). ${ }^{2}$ It usually occurs $2-12$ days after administration of chemotherapy. ${ }^{1}$ The initial symptoms are palmoplantar dysesthesia and tingling, which can evolve within a few days to burning pain and symmetric well-defined erythema with edematous swelling. In severe cases, blistering, desquamation and subsequent ulcera- tion can occur. ${ }^{1-3}$ It usually resolves within a few weeks after withdrawal of the responsible drug. ${ }^{1,3}$

The diagnosis of hand-foot syndrome is made based on its clinical features. The differential diagnosis includes other cutaneous drug reactions, erythromelalgia, graft-versus-host disease, chemotherapy-induced Raynaud syndrome and erythema multiforme.

Patients taking medications associated with hand-foot syndrome should be informed of the potential for development of this condition. Mechanical stress on the skin including pressure, friction and heat should be avoided. ${ }^{3}$ Regular use of moisturizing lotion may be helpful. Although various medications (e.g., pyridoxine, dimethylsulfoxide and oral corticosteroids) have been used to treat this syndrome, ${ }^{1}$ their use is not supported by randomized controlled trials.

\section{References}

1. Degen A, Alter M, Schenck F, et al. The hand-foot syndrome associated with medical tumor therapy — classification and management. J Dtsch Dermatol Ges 2010;8:652-61.

2. Lipworth AD, Robert C, Zhu AX. Hand-foot syndrome (handfoot skin reaction, palmar-plantar erythrodysesthesia): focus on sorafenib and sunitinib. Oncology 2009;77:257-71.

3. Webster-Gandy JD, How C, Harrold K. Palmar-plantar erythrodysesthesia (PPE): a literature review with commentary on experience in a cancer centre. Eur J Oncol Nurs 2007;11: 238-46. 